\title{
Faringoplastía de reposición con suturas barbadas como tratamiento quirúrgico en pacientes con síndrome de apnea-hipopnea obstructiva del sueño
}

\section{Barbed sutures reposition pharyngoplasty as surgical treatment in obstructive sleep apnea-hypopnea syndrome}

\author{
Felipe Castillo $\mathrm{F}^{1}$, Gianella Aceituno $\mathrm{B}^{2}$, Cristian Bachelet $\mathrm{R}^{3}$.
}

\begin{abstract}
RESUMEN
Introducción: La planificación de cirugías para el manejo del síndrome de apneahipopnea obstructiva del sueño (SAHOS) ha incrementado su precisión desde la introducción de la endoscopía del sueño inducido por fármacos (DISE).

Objetivo: Evaluar la técnica de faringoplastía de reposición con suturas barbadas (BRP) para el colapso velofaríngeo y/o de paredes laterales orofaríngeas evaluado mediante DISE en pacientes con SAHOS.

Material y método: Ochenta y ocho pacientes fueron evaluados para cirugía mediante antropometría, escala de somnolencia de Epworth (ESS) y poligrafía respiratoria. Veinte y seis de 88 pacientes fueron seleccionados. De los 26, 14 accedieron al tratamiento quirúrgico, el que se seleccionó en base a la DISE. En todos los casos, se realizó BRP.

Resultados: A los 3 meses de la cirugía hubo mejoría en 10/14 pacientes (criterios de Sher, disminución del índice de apnea-hipoapnea a <20 o 50\% del basal). La ESS bajó en promedio de 12 a 5 puntos $(p<0,05)$. No se reportaron incidentes en el posoperatorio y no han ocurrido eventos adversos.

Conclusión: La técnica de BRP es una técnica sencilla y útil para la expansión anterior y lateral del paladar blando y orofaringe, con una tasa de éxito similar en esta cohorte a la reportada internacionalmente.
\end{abstract}

Palabras clave: Síndromes de apnea del sueño, sutura barbada, faringoplastía.

\section{ABSTRACT}

Introduction: Surgical planning for the management of obstructive sleep apneahypopnea syndrome (OSAHS) has changed since the introduction of drug induced sleep endoscopy (DISE).

\footnotetext{
1 Servicio Otorrinolaringología, Unidad de Sueño Hospital de Puerto Montt y Clínica Universitaria de Puerto Montt. Facultad de Medicina Universidad San Sebastián - Sede Patagonia, Chile.

2 Hospital Clínico IST Viña del Mar, Universidad de Valparaíso, Chile.

3 Servicio Otorrinolaringología, Hospital Carlos van Buren. Facultad de Medicina Universidad de Valparaíso, Chile.

Los autores declaran no tener conflictos de interés.
}

Recibido el 11 de junio, 2019. Aceptado el 18 de enero, 2020. 
Aim: To evaluate the technique of barbed sutures reposition pharyngoplasty (BRP) for velopharyngeal collapse and/or oropharyngeal lateral walls after DISE evaluation in OSAHS patients.

Material and method: 88 patients were evaluated for surgery by anthropometry, Epworth sleepiness scale (ESS) and respiratory polygraphy. 26 of 88 patients were selected. Of the 26, 14 agreed to surgical treatment, which was selected on DISE findings. In all cases, BRP was performed.

Results: Three months after surgery there was improvement in 10/14 patients (Sher criteria, apnea-hypopnea index reduction at $<20$ or $50 \%$ of baseline). The ESS improved on average 12 to $5(p<0.05)$. No incidents were reported in the post-operative period and no adverse events were reported.

Conclusion: The BRP technique is a simple and useful technique for the anterior and lateral expansion of the soft palate and oropharynx, with a similar success rate in this cohort to that internationally reported.

Key words: Sleep apnea syndromes, barbed suture, pharyngoplasty.

\section{INTRODUCCIÓN}

El síndrome de apnea-hipoapnea obstructiva del sueño (SAHOS) es una patología altamente frecuente en la población ${ }^{1,2}$. Su asociación a patología cardiovascular, aumento de la accidentabilidad laboral e hipersomnia diurna está fuertemente documentada ${ }^{3-5}$, como también a otras comorbilidades como cáncer $^{6}$. A pesar de la importancia de este síndrome, en Chile sigue siendo una patología muy subdiagnosticada. En nuestro país, existen pocos trabajos que evalúen presencia de ronquido y SAHOS. Un reporte preliminar del proyecto PLATINO ${ }^{7}$, describe que la prevalencia de roncopatía en la población chilena es $66,4 \%$ ( $72 \%$ en hombres y $62 \%$ en mujeres). Según el mismo reporte, $34 \%$ de los hombres y $25 \%$ de mujeres tienen alto riesgo de SAHOS'.

El tratamiento de elección del SAHOS es la presión positiva continua sobre la vía aérea (CPAP). Este tratamiento es altamente efectivo entre quienes lo utilizan, sin embargo, 30\%-40\% de pacientes no utilizan el CPAP de manera adecuada. El tratamiento quirúrgico efectuado por el otorrinolaringólogo, es una alternativa para estos pacientes, con una efectividad aún discutible. Desde hace 10 años, la endoscopía del sueño inducido por fármacos (DISE) ha surgido como una herramienta eficaz en el estudio de pacientes candidatos a cirugía. Este estudio permite evaluar de forma dinámica los sitios de colapso en la vía aérea superior (VAS). En base al diagnóstico topográfico del colapso de la vía aérea, se puede entender cuáles son los sitios a tratar y actuar en consecuencia. La indicación de DISE en pacientes que serán sometidos a cirugía del sueño permite mejorar en la precisión diagnóstica de los sitios de colapso, aumentando la especificidad de la cirugía y mejorando sus resultados ${ }^{9-11}$.

\section{OBJETIVO}

En este artículo, evaluamos la eficacia de una nueva técnica de faringoplastía realizada con suturas barbadas para el tratamiento del colapso velofaríngeo y/o de paredes laterales orofaríngeas (PLO) evaluado mediante DISE. Esta técnica, que fue descrita el año 2015 por Vicini y cols, y se denomina faringoplastía de reposición con suturas barbadas $(\mathrm{BRP})^{12}$.

\section{MATERIAL Y MÉTODO}

Ochenta y ocho pacientes con historia de roncopatía y alta probabilidad pretest de SAHOS (obtenida mediante cuestionario STOP-BANG) evaluados durante 2016 en una consulta privada fueron revisados retrospectivamente para efectos de incluirlos en este artículo. Todos firmaron un consentimiento informado accediendo a utilizar sus datos para eventuales publicaciones. Cada paciente evaluado en consulta es incluido en una base de datos donde se incluyen las siguientes variables: circunferencia cervical (CC); peso, estatura e índice de masa corporal (IMC); 
puntaje de Friedman (FS) (combinando Mallampati modificado, tamaño de amígdalas y su asociación a IMC); escala de somnolencia de Epworth (ESS) para evaluar somnolencia diurna; y resultados de poligrafía respiratoria (PG) (Polígrafo BMC YH-600A PolyWatch $^{\circledR}$ ) para medición de índice de apneahipoapnea (IAH) e índice de desaturación de oxígeno (ID0).

Se sugirió cirugía a aquellos pacientes con falla, rechazo 0 no indicación categórica de uso de CPAP. Se considera falla en caso de mala adherencia (uso menor a 4 horas al día y 5 días a la semana de seguimiento en los últimos 3 meses) o intolerancia a CPAP, así como rechazo a la decisión expresa del paciente a no querer usar el dispositivo. Por otro lado, la indicación categórica de uso de CPAP se da en pacientes con IAH de al menos 15 $15^{13}$. En todos estos grupos de pacientes mencionados, se consideraron candidatos quirúrgicos a quienes tuvieran una CC menor a 46 $\mathrm{cm}$, IAH menor a 60 y un FS menor a 4 (Tabla 1).

Todos los pacientes quirúrgicos firmaron un consentimiento informado de DISE y de cirugía incluyendo cirugía nasal (septoplastía y turbinoplastía), así como de eventual amigdalectomía con faringoplastía y cirugía de base de lengua 0 epiglotis según los resultados de la DISE. EI consentimiento informado de cirugía incluyó la posibilidad de falla terapéutica, infección, sangrado, compromiso de vía aérea y eventuales complicaciones durante la DISE.

La DISE se realizó previo al inicio de la anestesia y fue evaluada según escala VOTE de estadificación ${ }^{14}$. Se les indicó a los pacientes que se les inter- vendría máximo 3 niveles de colapso en esta cirugía, dejando para un segundo tiempo el cuarto 0 quinto nivel de colapso en caso de encontrarlo, por motivos de manejo del dolor posoperatorio. La DISE se realizó con dexmedetomidina (Precedex ${ }^{\circledR}$, Pfizer-USA) a $2 \mu \mathrm{g} / \mathrm{kg} / \mathrm{h}$ los primeros 20 minutos, bajando la dosis a $1 \mu \mathrm{g} / \mathrm{kg} / \mathrm{h}$ los siguientes minutos hasta lograr una sedación y ronquido en un plano clínicamente estable (escala de sedación de Ramsay en 5). Todas las DISE fueron grabadas en dispositivos de video para su posterior revisión. Tanto las DISE como las cirugías fueron realizadas en Clínica Universitaria de Puerto Montt. Todas las DISE se realizaron inmediatamente antes de cada cirugía.

Se consideró un éxito quirúrgico a aquellos pacientes que alcanzaran en posoperatorio a los 3 meses los criterios descritos por Sher: disminución del IAH a $<20$ y reducción de al menos 50\% del IAH basal' ${ }^{15}$. La medición del IAH a los 3 meses se realizó con el mismo equipo poligráfico que se usó para el diagnóstico, momento en el cual se volvió a realizar ESS y medición de peso para cálculo de IMC. Las BRP fueron realizadas posteriores a amigdalectomía dejando expuesto el músculo palatofaríngeo (MPF) (Figura 1).

\section{Tabla 1. Criterios de inclusión para candidatos} quirúrgicos

Circunferencia cervical menor a $46 \mathrm{cms}$

IAH menor a 60

Score de Friedman menor a 4

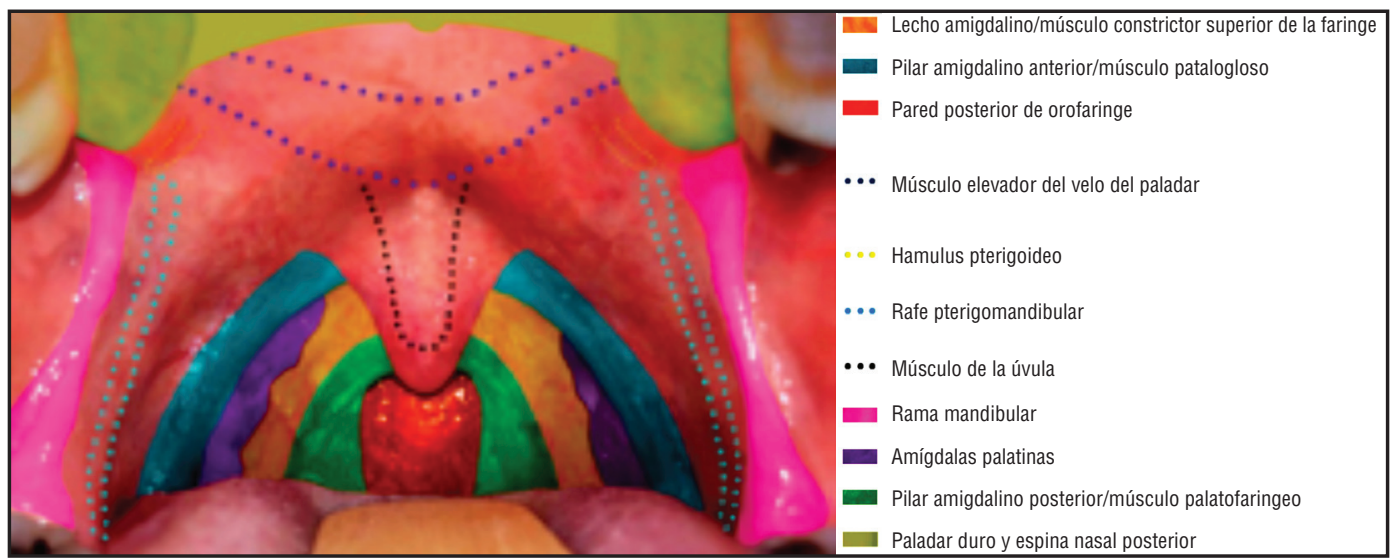

Figura1. Anatomía orofaríngea. 
En caso de colapso anteroposterior o concéntrico del paladar se utilizó sutura barbada Stratafix ${ }^{\circledR}$ (Ethicon-USA) 2-0 con fuerza tensil 3-0 y agujas 26 ó 36 mms con punta redonda 0 triangular según las características del paladar del paciente. Se realizó una sección de la cúpula del palatogloso para una mejor exposición del lecho amigdalar. Una vez realizado esto, se identificó el MPF para seccionarlo transversalmente dejando el cabo inferior anudado con sutura hemostática con Vicryl 3-0 (Ethicon-USA). La colocación de la sutura barbada se realizó pasando la aguja desde la espina nasal posterior diseñando 4 "vectores de tensión", uno con esquina en la zona superior de la fosa amigdalar y entre la espina nasal posterior y hamulus pterigoideo, y 3 uniendo el músculo palatofaríngeo previamente seccionado y el rafe pterigomandibular (Figura 2).

El punto de tensión de este vector a nivel superior de la fosa amigdalar se eligió en base a los hallazgos de la DISE. En caso de colapsos largos y concéntricos, el punto de tensión fue colocado muy cercano a la fosa amigdalar (5 a 10 milímetros) y en una zona más lateral. En caso de colapsos anteroposteriores y cortos, el punto de tensión fue colocado 10 a 20 milímetros sobre la fosa amigdalar y en un punto más central. En caso de encontrar colapsos a nivel de PLO, se realizó esta misma técnica, realizando amigdalectomía previa y luego seccionando a nivel medio el MPF, para posteriormente subir este músculo en dirección al rafe pterigomandibular. Para colapsos a nivel de la base de lengua 0 epiglotis, en caso de existir, las intervenciones a este nivel se dejaron para un segundo tiempo. En caso de que los pacientes requirieran cirugía nasal, se realizó una septoplastía y/o turbinoplastía.

\section{RESULTADOS}

De los 88 pacientes incluidos en el estudio, 26 calificaron por anatomía como candidatos favorables para manejo quirúrgico. De estos 26 pacientes, 14 firmaron los consentimientos de cirugía y DISE y prefirieron finalmente cirugía por sobre la opción del CPAP (Tabla 2).

Estos 14 pacientes fueron incluidos en un protocolo de seguimiento de cirugía multinivel en uno o dos tiempos, incluyendo en esta cohorte los resultados del primer tiempo quirúrgico. Se les ex-

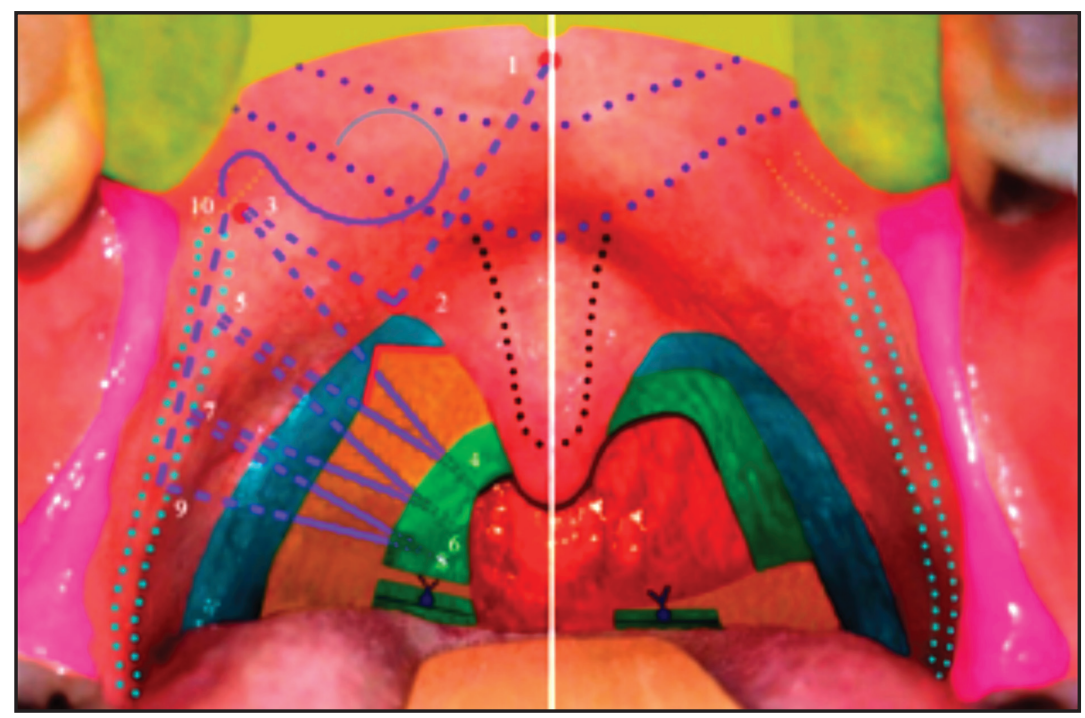

Figura 2. Faringoplastía de reposición con suturas barbadas. La aguja de Stratafix comienza pasándose desde la espina nasal posterior (1), saliendo a nivel de la cúpula del lecho amigdalar (2). Se vuelve a entrar en este mismo punto (2) saliendo a nivel del hammulus pterigoideo (3), conformando el primer vector de tensión. Desde este punto se entra otra vez (3) saliendo a nivel del MPF a nivel superior (4) y desde aquí (4) se entra nuevamente saliendo a nivel del rafe pterigomandibular (5), conformando tres vectores de tensión entre los puntos 3-4-5, 5-6-7 y 7-8-9. Se finaliza el paso de la aguja desde el punto 9 saliendo nuevamente a nivel del hammulus pterigoideo (10). Previamente, el MPF se seccionó medialmente, colocando una sutura de vicryl a nivel del polo inferior del MPF. 
Tabla 2. Características de los pacientes seleccionados para cirugía

\begin{tabular}{|cccccccc|}
\hline $\mathrm{n}$ & Género & Edad & CC & IMC & Friedman & ESS & IAH preop. \\
\hline 1 & M & 34 & 38 & 26 & 2 & 7 & 5,3 \\
2 & $\mathrm{M}$ & 54 & 46 & 30 & 3 & 10 & 29,5 \\
3 & $\mathrm{M}$ & 46 & 37 & 24 & 2 & 17 & 41,1 \\
4 & $\mathrm{M}$ & 33 & 45 & 39 & 3 & 14 & 13,1 \\
5 & $\mathrm{M}$ & 38 & 41 & 27 & 3 & 15 & 52,7 \\
6 & $\mathrm{~F}$ & 37 & 35 & 23 & 2 & 12 & 22 \\
7 & $\mathrm{M}$ & 33 & 41 & 28 & 3 & 18 & 16,8 \\
8 & $\mathrm{M}$ & 41 & 42 & 29 & 2 & 9 & 11,2 \\
9 & $\mathrm{~F}$ & 35 & 35 & 25 & 2 & 15 & 37,2 \\
10 & $\mathrm{M}$ & 41 & 37 & 29 & 3 & 22 & 32 \\
11 & $\mathrm{~F}$ & 45 & 40 & 27 & 3 & 5 & 57,8 \\
12 & $\mathrm{M}$ & 59 & 41 & 25 & 3 & 8 & 57,7 \\
13 & $\mathrm{M}$ & 43 & 44 & 30 & 3 & 14 & 15 \\
14 & $\mathrm{M}$ & 36 & 44 & 34 & 3 & 7 & 37,4 \\
\hline
\end{tabular}

$\mathrm{n}=$ Paciente. $\mathrm{M}=$ =Masculino, F =Femenino. CC =Circunferencia cervical. IMC =Índice de masa corporal en kg/m². IAH: Índice de apnea-hipopnea.

plicó a los pacientes que justo antes de la cirugía, serían sometidos a una DISE que permitiría saber qué lugares de colapso influyen en su condición de SAHOS. Todos los pacientes presentaban algún tipo de obstrucción nasal, por lo que todos fueron sometidos a septoplastía y/o turbinoplastía, considerando éste el primer nivel intervenido. Se consideró velofaringe como el nivel 2, PLO como nivel 3 , base de lengua como nivel 4 y epiglotis como nivel 5. En la DISE, 3 pacientes tuvieron un colapso anteroposterior a nivel velofaríngeo, 2 completo y 1 parcial. Once pacientes tuvieron colapso velofaríngeo concéntrico, 9 completo y 2 parcial. A nivel de PLO, hubo 5 pacientes con colapso completo,
7 parcial y 2 sin colapso orofaríngeo. Hubo 3 casos con colapso de base de lengua en la DISE y 1 colapso epiglótico, los que esperan resolución quirúrgica en un segundo tiempo (Tabla 3).

En todos los casos, se realizó BRP (Stratafix, Ethicon ${ }^{\circledR}$, EE.UU) con variaciones según la DISE y según lo expuesto previamente. Se obtuvo criterios de éxito quirúrgico a los 3 meses posoperatorios en $10 / 14$ pacientes $(71,4 \%)$. Excluyendo casos con colapso de base de lengua-epiglotis y dejando sólo aquellos con colapso velofaríngeo-PLO, se obtuvo éxito en $8 / 10$ pacientes (80\%). Al excluir de los casos sólo a los pacientes con colapso de base de lengua, 9/11 lograron éxito (81,9\%) (Figura 3). La

Tabla 3. Tipos de colapso de los pacientes según escala VOTE

\begin{tabular}{|c|c|c|c|c|}
\hline$n$ & V & 0 & $\mathrm{~T}$ & $E$ \\
\hline 1 & $2 c$ & 2 & 0 & 0 \\
\hline 2 & 2ap & 0 & 1 & 0 \\
\hline 3 & $2 c$ & 2 & 0 & 0 \\
\hline 4 & $2 c$ & 1 & 0 & 0 \\
\hline 5 & $1 \mathrm{c}$ & 1 & 0 & 0 \\
\hline 6 & $2 c$ & 2 & 0 & 0 \\
\hline 7 & $2 c$ & 1 & 1 & 0 \\
\hline 8 & $1 \mathrm{c}$ & 1 & 0 & 0 \\
\hline 9 & 1ap & 0 & 0 & 1 \\
\hline 10 & 1ap & 1 & 0 & 0 \\
\hline 11 & $2 c$ & 2 & 0 & 0 \\
\hline 12 & $2 c$ & 1 & 0 & 0 \\
\hline 13 & $2 C$ & 1 & 1 & 0 \\
\hline 14 & $2 c$ & 2 & 0 & 0 \\
\hline
\end{tabular}




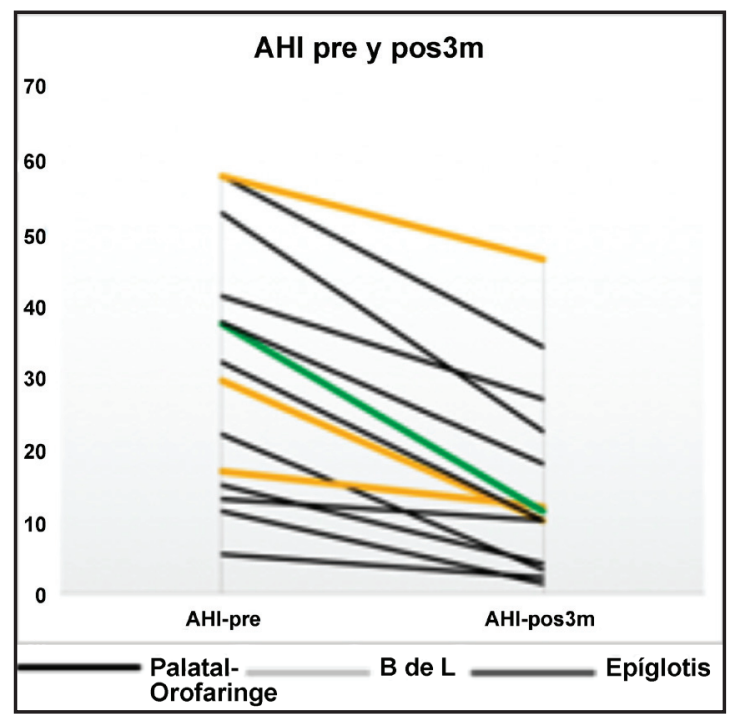

Figura 3. Mejoría del IAH a los 3 meses de la cirugía.

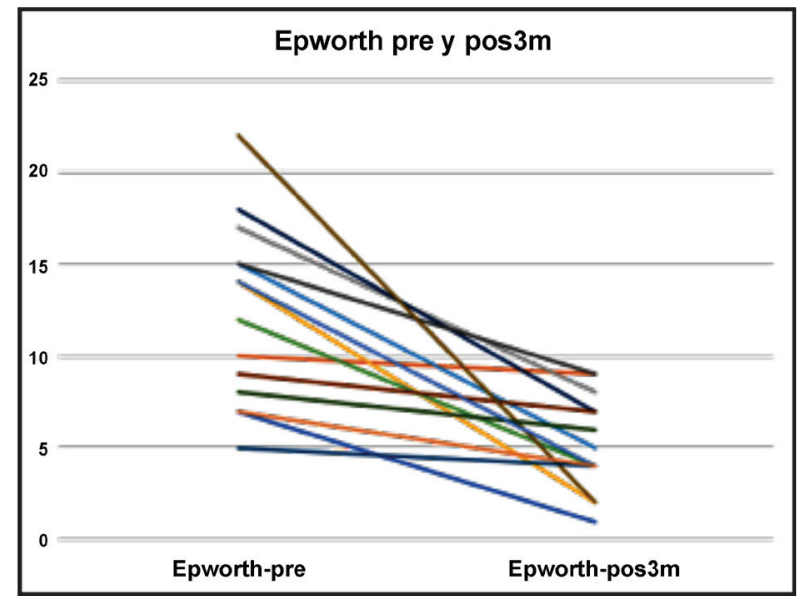

Figura 4. Mejoría del ESS a los 3 meses de la cirugía.

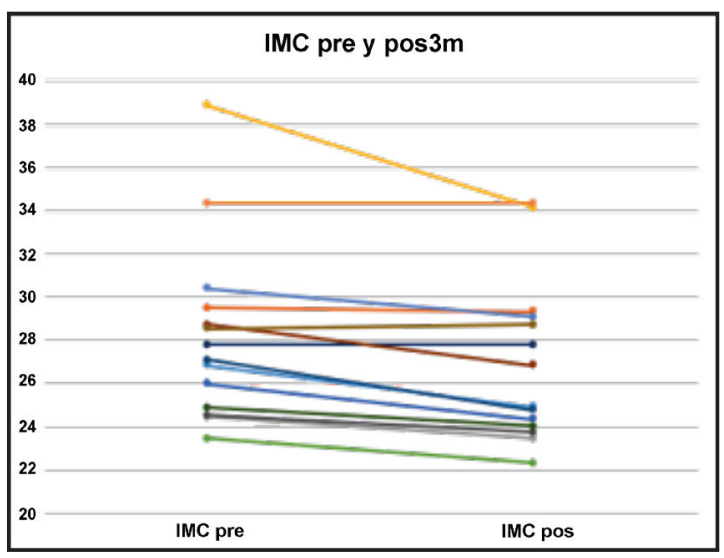

Figura 5. Mejoría del IMC a los 3 meses de la cirugía. 
ESS mejoró en promedio de 12 a $5(p<0,05)$. El peso bajó de $84,4 \mathrm{~kg}$ a $81 \mathrm{~kg}$ a los 3 meses de la cirugía ( $p<0,05)$, sin embargo al comparar el IMC promedio, la diferencia pre y posoperatoria no fue significativa (28 a $27,5 \mathrm{~kg} / \mathrm{mt}^{2}, \mathrm{p}>0,05$ ) (Figuras 4 y 5$)$. No se reportaron incidentes en el posoperatorio ni eventos adversos hasta un año después de cada cirugía.

\section{DISCUSIÓN}

Entre los tratamientos del SAHOS, el desarrollo de cirugía del sueño ha tenido un resurgimiento, ya sea como alternativa principal de tratamiento o como complemento para adaptación a CPAP. Esto ha sido posible gracias al análisis detallado de los pacientes mediante el topo-diagnóstico del colapso de la VAS, lo que ha permitido una selección más precisa de técnicas quirúrgicas para cada paciente y así romper el anterior paradigma de que una misma técnica quirúrgica era aplicable para todos los casos.

Gracias a la sistematización del topo-diagnóstico del colapso dinámico de los tejidos blandos de la VAS mediante nasofibroscopía sensibilizada y DISE, se ha logrado identificar diferentes zonas en la VAS para su manejo: velofaringe, paredes laterales faríngeas, base de lengua y epiglotis. En particular, las intervenciones sobre el velo del paladar y las paredes laterales faríngeas (principalmente las PLO), han tenido una gran evolución con la aparición de nuevas técnicas de faringoplastía que pretenden expandir y aumentar tensión de forma menos mutilante, con mejores resultados y más funcionales. Existe una gran variedad de faringoplastías descritas en la literatura. No obstante, al hacer un análisis detallado del enfoque anatómico de ellas, podríamos agruparlas en aquellas orientadas principalmente a la línea media palatina (LMP) y aquellas orientadas principalmente a la reposición del MPF.

Las "cirugías de reposición del MPF", son técnicas enfocadas principalmente al manejo de colapsos laterales velofaríngeos (aunque aplicables en otras formas de colapso a este nivel) y de las PLO. Como principio básico incluyen la disección del MPF y liberación de su inserción más inferior, para permitir la creación de un colgajo de este músculo. Esto permite su adecuada reposición con menor tensión, logrando una mejor estabilidad. Este principio fue originado por Cahali cuando describió su faringoplastía late$\mathrm{ral}^{16}$. A partir de este concepto, se crearon también otras técnicas reconocidas hoy en día como la faringoplastía de expansión de esfínter (ESP) de Pang y Woodson ${ }^{17}$, la faringoplastía de recolocación de $\mathrm{Li}^{18} \mathrm{y}$ la misma BRP. La BRP es una de las últimas técnicas de reposición del MPF ampliamente difundida a nivel mundial. Esta técnica mostró buenos resultados iniciales en experiencias tanto como cirugía exclusiva ${ }^{12}$, así también como cirugía multinivel con reducción de base de lengua transoral robótica (TORS) ${ }^{19}$.

La BRP se considera un resultado de la combinación de varios principios que han constituido la evolución de la faringoplastía, a lo que se conoce el día de hoy. Otro concepto incorporado en algunas faringoplastías de reposición del MPF y que ha sido especialmente difundido por MacKay ${ }^{20}$, es la resección de grasa supratonsilar para mejorar el espacio a nivel de la cúpula palatina. Esto se realiza a través de la resección de un triángulo mucoso en la parte superior del músculo palatogloso. De este modo, al realizar una tracción del MPF con sutura en la unión de tu tercio superior con su tercio medio, permite su reposicionamiento hacia el borde anterior del paladar blando, dando una gran expansión en diámetro transverso de la vía aérea a este nivel, con una faringe de aspecto más cuadrado. A este cambio de posición del MPF también se le ha llamado "robo del pilar posterior" (en analogía a la técnica de robo de crura lateral del cartílago alar en rinoplastía), el cual permite una posición final del MPF más lateral y anterior, mejorando no sólo el diámetro transverso orofaríngeo, sino también el espacio retropalatal en sentido anteroposterior ${ }^{12}$.

La BRP incorpora también la mejoría de la estabilidad anatómica de las suturas, al utilizar de anclaje ciertas regiones de alta densidad fibrosa como son la espina nasal posterior, el hamulus pterigoídeo y el rafe pterigomandibular, los cuales son ubicados en el intraoperatorio por inspección y palpación. Esto está basado en principios descritos por Mantovani y cols en su técnica original de faringoplastía de cortinas romanas o "roman blinds technique" (RBT) orientada inicialmente al manejo de la LMP principalmente ${ }^{21}$. Finalmente, incorpora además el uso de suturas barbadas unidireccionales reabsorbibles, basado en lo descrito por el grupo de Mantovani, quien modificó su técnica RBT usando estas suturas. Éstas permiten una manipulación mucho más fácil, sin necesidad 
de realizar nudos, generando menos daño a los tejidos y acortando el tiempo quirúrgico ${ }^{22,23}$.

En un estudio se compararon diferentes cirugías de paladar como terapia exclusiva: uvulopalatofaringoplastía (UPPP) modificada por Fairbanks, ESP y BRP. Este estudio mostró que la reducción posoperatoria del IAH y ESS fue significativamente mayor en BRP, comparado con ESP y UPPP. La reducción del IDO no tuvo diferencias entre BRP y ESP, pero en ambas fue mayor que con UPPP. Por último, la saturación mínima de oxígeno posoperatoria no tuvo una mejoría significativa con ninguna de las tres técnicas ${ }^{24}$. En otro estudio donde se compararon diferentes cirugías de paladar (UPPP, ESP y BRP) en contexto de cirugía multinivel con TORS, se encontró que BRP y ESP no tuvieron diferencias significativas en cuanto a éxito quirúrgico, pero ambas fueron mejores que UPPP. No obstante, sí hubo diferencia respecto al tiempo quirúrgico, siendo la BRP más rápida de ejecutar que las otras técnicas ${ }^{25}$.

Otro estudio que propuso un nuevo instrumento de evaluación de problemas posoperatorios en cirugía de paladar, probó este cuestionario en pacientes operados con BRP y ESP. Al comparar ambas técnicas, el cuestionario demostró mayor severidad en percepción de disfagia y cambios en sensibilidad faríngea en la ESP respecto a BRP26. El estudio multicéntrico más grande existente con BRP, cuenta con 111 casos manejados como cirugía exclusiva ( $94,6 \%$ de los casos, incluyendo cirugía nasal o amigdalectomía como asociados) 0 en contexto multinivel (con reducción de base de lengua 0 suspensión de hioides). Este estudio demostró una reducción del IAH desde 33,4 $\pm 19,5$ a $13,5 \pm 10,3$ con un éxito quirúrgico de $73 \%$. Además, se observó una reducción del ID0 desde 29,6 $\pm 20,7$ a $12,7 \pm 10,8$ y de la ESS de $10,2 \pm 4,5$ a $6,1 \pm 3,6$. Se observaron complicaciones intraoperatorias en $7 \%$ de los casos (principalmente extrusiones de sutura y sangrado) y posoperatorias en $32 \%$ de los casos (principalmente disfagia transitoria resuelta a la semana después de la intervención) ${ }^{27}$.

Ha sido tal el impacto de las suturas barbadas en faringoplastías, que además de la RBT de Mantovani y la BRP de Vicini han aparecido una serie de modificaciones de éstas y otras técnicas para el manejo de LMP y PLO. Salamanca y cols describieron una modificación con sutura barbada de la palatoplastía anterior de Pang para manejo de la LMP28. Mantovani y cols describieron la "técnica de la Alianza", que modifica la RBT para mejorar su manejo de las PLO29. Pianta y $\operatorname{cols}^{30}$ y Sorrenti y cols ${ }^{31}$ presentaron diferentes modificaciones con sutura barbada de la ESP para manejo de PLO. Finalmente, Barbieri y cols describieron la faringoplastía de suspensión barbada, que modifica la BRP para mejorar su manejo de la LMP32. En este estudio, existen limitaciones de la metodología que son difíciles de soslayar y forman parte de la práctica habitual en cirugía y medicina del sueño. Una de ellas es el uso de PG en lugar de polisomnografía (PSG) como estudio diagnóstico y de control de éxito quirúrgico. La PSG es el examen de elección para el diagnóstico de SAHOS, sin embargo tanto para diagnóstico como seguimiento, su uso está limitado por el costo y acceso a este examen. A pesar de esto, está ampliamente validada la $P G$ como estudio alternativo en casos con alta probabilidad pretest de SAHOS, I0 cual se verificó en la selección de los pacientes de este trabajo $0^{33}$. Otra dificultad en un estudio como este es lograr la evaluación del efecto aislado de una cirugía respecto de otras cirugías concomitantes, ya que habitualmente las faringoplastías son planeadas en contexto de "cirugía multinivel de tejidos blandos de la VAS". En este estudio en concreto, las BRP se asociaron a intervenciones a nivel nasal, dejando otras a nivel de base de lengua y epiglotis para otro tiempo quirúrgico. Es esperable que el impacto en el IAH de la cirugía nasal como único complemento a la BRP no sea de gran magnitud, ya que es sabido que, a pesar de ser muy importante en la dinámica de la VAS, la cirugía nasal como terapia exclusiva no genera una reducción importante en el IAH, pero sí en la intensidad del ronquido, calidad subjetiva de sueño y ESS ${ }^{34}$.

El volumen amigdalino suele ser otro factor a considerar en el éxito quirúrgico de la cirugía de partes blandas en SAHOS. Friedman y cols ${ }^{35}$, mostraron que pacientes con amígdalas grandes y una relación paladar-lengua favorable medido según Mallampati modificado, era un factor predictor de éxito quirúrgico en pacientes sometidos a uvulopalatofaringoplastía tradicional (UPPP). La amigdalectomía aislada también puede ser una alternativa en el manejo de los pacientes SAHOS. Senchak y cols $^{36}$ sometieron a 19 pacientes con SAHOS moderado y amígdalas grado III a amigdalectomía, reportando una baja del IAH de 18 a 3,2 después de 2-6 meses de la cirugía. En nuestro estudio, plantear una amigdalectomía exclusiva en pacientes con amígdalas de diversos tamaños (Tabla 2) 
habría sido quizás infructuoso. El DISE permite determinar cuáles son los puntos de colapso de la vía aérea y actuar en consecuencia. En nuestra cohorte, encontramos un colapso velofaríngeo en la totalidad de los casos, por lo que consideramos mandatorio realizar alguna técnica en paladar con énfasis además en la PLO en los casos en donde además encontramos colapso a este nivel (5/14 casos).

\section{BIBLIOGRAFÍA}

1. Young T, Palta M, Dempsey J, Skatrud J, Weber $S$, BADR S. The occurrence of sleep-disordered breathing among middle-aged adults. N Engl J Med 1993; 328: 1230-35.

2. Duran J, Esnaola S, Rubio R, Iztueta A. Obstructive sleep apnea-hypopnea and related clinical features in a population-based sample of subjects aged 30 to 70 yr. Am J Respir Crit Care Med 2001;163: 685-9.

3. Barbe F, Duran-Cantolla J, Sanchez-de-la-Torre $M$, ET AL. Effect of continuous positive airway pressure on the incidence of hypertension and cardiovascular events in nonsleepy patients with obstructive sleep apnea: a randomized controlled trial. JAMA 2012; 307: 2161-8.

4. Gottlieb DJ, Yenokyan G, Newman AB, et al. Prospective study of obstructive sleep apnea and incident coronary heart disease and heart failure: the sleep heart health study. Circulation 2010; 122: 352-60.

5. Teran-Santos J, Jiménez-Gómez A, Cordero-Guevara J. The association between sleep apnea and the risk of traffic accidents. Cooperative Group BurgosSantander. N Engl J Med 1999; 340: 847-51.

6. Nieto FJ, Peppard PE, Young T, Finn L, Hla KM, Farre R. Sleep-disordered breathing and cancer mortality: results from the Wisconsin Sleep Cohort Study. Am J Respir Crit Care Med 2012; 186: 190-4.

7. Proyecto Latinoamericano de Investigación en Obstrucción Pulmonar (PLATINO) http://www. platino-alat.org/

8. Jorquera J, Valdivia G, Pertuzé J, Dreyse J, Lisboa C. Prevalencia de ronquido y de síntomas relacionados a apnea obstructiva del sueño en la población chilena mayor de 40 años: Comunicación preliminar-proyecto platino. Departamentos de Enf. Respiratorias y Salud

\section{CONCLUSIÓN}

Nuestro estudio presenta resultados comparables con lo descrito en la literatura internacional. Se puede inferir que estos resultados son favorables gracias a una adecuada selección de los pacientes donde se incorpora el DISE como método de topo-diagnóstico dinámico del colapso de tejidos blandos de la VAS.

Pública. Pontificia Universidad Católica de Chile.

9. Koutsourelakis I, Safiruddin F, Ravesloot M, Zakynthinos S, de VRies N. Surgery for obstructive sleep apnea: sleep endoscopy determinants of outcome. Laryngoscope 2012; 122: 2587-91.

10. KEZIRIAN EJ. Nonresponders to pharyngeal surgery for obstructive sleep apnea: insights from druginduced sleep endoscopy. Laryngoscope 2011; 121: 1320-6.

11. Soares D, Sinawe H, Folbe AJ et al. Lateral oropharyngeal wall and supraglottic airway collapse associated with failure in sleep apnea surgery. Laryngoscope 2012; 122: 473-9.

12. Vicini C, Hendawy E, Campanini A, et al. Barbed reposition pharyngoplasty (BRP) for OSAHS: a feasibility, safety, efficacy and teachability pilot study. "We are on the giant's shoulders". Eur Arch Otorhinolaryngol 2015; 272: 3065-70.

13. LJ Epstein, D Kristo, PJ Strollo et al. Clinical Guideline for the Evaluation, Management and Longterm Care of Obstructive Sleep Apnea in Adults. Journal of Clinical Sleep Medicine 2009; 5: 263-76.

14. Hohenhorst W, Ravesloot MJL, Kezirian EJ, et AL. Drug-induced sleep endoscopy in adults with sleep-disordered breathing: Technique and the VOTE classification system. Operative Techniques in Otolaryngology 2012; 23: 11-8.

15. Sher AE, Schechtman KB, Piccirillo JF. The efficacy of surgical modifications of the upper airway in adults with obstructive sleep apnea syndrome. Sleep 1996; 19: 156-77.

16. CahAl MB. Lateral pharyngoplasty: a new treatment for obstructive sleep apnea hypopnea syndrome. Laryngoscope 2003; 113: 1961-8.

17. Pang KP, Woodson BT. Expansion sphincter pharyngoplasty: a new technique for the treatment of obstructive sleep apnea. Otolaryngol Head Neck Surg 2007; 137: 110-4. 
18. LI HY, LeE LA. Relocation pharyngoplasty for obstructive sleep apnea. Laryngoscope 2009; 119: 2472-7.

19. Vicini C, Meccariello G, Cammaroto G, et al. Barbed reposition pharyngoplasty in multilevel robotic surgery for obstructive sleep apnoea. Acta Otorhinolaryngol Ital 2017; 37: 214-7.

20. MacKay SG, Carney AS, Woods C, et al. Modified uvulopalatopharyngoplasty and coblation channeling of the tongue for obstructive sleep apnea: a multi-centre australian trial. J Clin Sleep Med 2013; 9: 117-24.

21. Mantovanı M, Minetti A, Torretta S, et al. The velo-uvulo-pharyngeal lift or "roman blinds" technique for treatment of snoring: a preliminary report. Acta Otorhinolaryngol Ital 2012; 32: 4853.

22. Mantovani M, Minetti A, Torretta S, et al. The "barbed roman blinds" technique: a step forward. Acta Otorhinolaryngol Ital 2013; 33: 128.

23. Mantovani M, Rinaldi V, Torretta S, et al. Barbed roman blinds technique for the treatment of obstructive sleep apnea: how we do it? Eur Arch Otorhinolaryngol 2016; 273: 517-23.

24. Rashinan MS, Montevecchi F, Cammaroto G, et al. Evolution of soft palate surgery techniques for obstructive sleep apnea patients: A comparative study for single-level palatal surgeries. Clin Otolaryngol 2018; 43: 584-90.

25. Cammaroto G, Montevecchi F, D’Agostino G, et al. Palatal surgery in a transoral robotic setting (TORS): preliminary results of a retrospective comparison between uvulopalatopharyngoplasty (UPPP), expansion sphincter pharyngoplasty (ESP) and barbed repositioning pharyngoplasty (BRP). Acta Otorhinolaryngol Ital 2017; 37: 4069.

26. Rashiman MS, Montevecchi F, Firinua E, et al. Let's know from our patients: PPOPS score for palate surgery evaluation/a pilot study. Eur Arch Otorhinolaryngol 2018; 275: 287-91.
27. Montevecchi F, Meccariello G, Firinu E, et al. Prospective multicentre study on barbed reposition pharyngoplasty standing alone or as part of multilevel surgery for sleep apnoea. Clin Otolaryngol 2018; 43: 483-8.

28. Salamanca F, Costantinı F, Mantovani M, et al. Barbed anterior pharyngoplasty: an evolution of anterior palatoplasty. Acta Otorhinolaryngol Ital 2014; 34: 434-8.

29. Mantovani M, Carioli D, Torretta S, et al. Barbed snore surgery for concentric at the velum collapse: the Alianza technique. $J$ Craniomaxillofac Surg 2017; 45: 1794-800.

30. Pianta L, Bertazzoni G, Morello R, et al. Barbed expansion sphincter pharyngoplasty for the treatment of oropharyngeal collapse in OSAS: a retrospective study on 17 patients. Clin Otolaryngol 2018; 43: 696-700.

31. Sorrenti G, Pelligra I, Albertini R, et al. Functional expansion pharyngoplasty: technical update by unidirectional barbed sutures. Clin Otolaryngol 2018; 43: 1419-21.

32. Barbieri M, Missale F, Incandela F, et al. Barbed suspension pharyngoplasty for treatment of lateral pharyngeal wall and palatal collapse in patients affected by OSAHS. Eur Arch Otorhinolaryngol 2019; 276: 1829-35.

33. Kapur VK, Auckley DH. ChowdhuRI S, et AL. Clinical practice guideline for diagnostic testing for adult obstructive sleep apnea: An American Academy of Sleep Medicine clinical practice guideline. $J$ Clin Sleep Med 2017, 13: 479-504.

34. JoHnson DM, SOOSE RJ. Updated nasal surgery for obstructive sleep apnea. Adv Otorhinolaryngol 2017; 80: 66-73.

35. Friedman M, Ibrahim $H$, Bass L. Clinical staging for sleep-disordered breathing. Otolaryngol Head Neck Surg 2002; 127: 13-21.

36. Senchak A, McKinlay A, Acevedo J et al. The Effect of Tonsillectomy Alone in Adult Obstructive Sleep Apnea. Otolaryngol Head Neck Surg 2015; 152: 969-73. 